\title{
MEMS sensor-based monitoring system for engineered geological disposal facilities
}

\author{
Wenbin Yang*, Rebecca Lunn, Alessandro Tarantino \\ Department of Civil and Environmental Engineering, University of Strathclyde \\ *Corresponding author e-mail: wenbin.yang @ strath.ac.uk
}

\begin{abstract}
The design of a novel MEMS (Micro-Electro-Mechanical System) sensor-based monitoring system is presented in this article for the in-situ monitoring of the conditions (temperature, relative humidity) of an engineered bentonite barrier for the underground disposal of radioactive waste in a geological disposal facility (GDF). A first prototype of the monitoring system incorporating multiple state-ofthe-art MEMS sensors has been developed on a PCB-based (Printed Circuit Board) structure, in order to measure the variation of temperature and relative humidity inside a cylindrical bentonite block during the hydration process. The monitoring system comprises of separate sensor boards, the microcontroller-equipped interface board, and the software user interface in Labview environment. One of the main design priorities is to reduce the size of the embedded sensors in order to minimize their influence on the hydro-mechanical response of the bentonite block. The sensor boards are encapsulated in different manners to protect them from moisture, chemical corrosion and mechanical shocks. The sensor system has been tested and calibrated in the temperature range between $-20^{\circ} \mathrm{C}$ and $120^{\circ} \mathrm{C}$, and at different relative humidity levels implemented by saturated salt solutions in enclosed containers. Test results demonstrate that the sensors have shown good functionality and robustness in harsh test environments such as high temperature and high humidity. Both temperature and relative humidity sensors have shown
\end{abstract}


satisfactory precision level and temporal stability, which are in good accordance with the design specification of these devices.

\section{Introduction}

The real-time monitoring of deep geological disposal facilities (GDFs) for radioactive waste disposal has attracted increasing research interest in recent years. Many countries are seeking long-term monitoring technologies that can function reliably over the operational timescales of a GDF: this may be in excess of 100 years (Lidskog et al. 2002). A key component of any monitoring system will be the in-situ monitoring of the thermo-hydro-mechanical-chemical (THMC) properties of bentonite in the engineered barrier system (EBS); the EBS plays a key role in safety arguments that ensure the long-term isolation of radionuclides from the nearsurface environment.

Fig. 1 shows part of the KBS-3V concept for the deep geological disposal of highlevel nuclear waste in crystalline rock, which was developed by SKB, Sweden. The compacted bentonite buffer relies on multiple THMC properties to ensure longterm safety of the system. It must generate a swelling pressure of between 2 and 10 MPa for hydraulic sealing and to minimise microbial activity. It must also ensure that transport through the EBS is via diffusion only and that the EBS can undergo sufficiently large plastic deformations so as to protect the waste canister from structural damage due to low-magnitude seismic events (Juvankoski et al. 2012).

This research, conducted under the framework of the multi-partner SAFE Barriers Project, focuses on the design of a MEMS-based (Micro-Electro-Mechanical System) sensor for the simultaneous monitoring of temperature and humidity 
within, or adjacent to, the compacted bentonite buffer in the EBS. Improved monitoring of bentonite will not only fulfil research and development needs, but will also be a requirement of the regulators during GDF operation. The GDF implementer will need to demonstrate that model calculations used to predict future behaviour in the operational safety case are reliable.

\section{Background}

The objectives of this research are to identify suitable commercial MEMS sensors for simultaneous monitoring of temperature and humidity, to develop a MEMSbased monitoring device for use in subsurface environments, and to evaluate sensor reliability and accuracy over the range of values likely to be encountered within the bentonite barrier. Historically, an extensive range of sensors has been deployed in radioactive waste disposal facilities and in underground testing laboratories over the past decades (Alonso et al. 2009; Breen et al. 2012). While the measurement principle of the sensors varies, one common restraint of these traditional sensors lies in the unit size of the sensor (typically in the order of $10 \mathrm{~cm}$ ), which limits the spatial resolution of the sensing device. A primary challenge is to reduce the dimensions of the sensor to a minimum, in order to improve the spatial resolution of the data, especially in locations that may have high thermal and/or humidity gradients. In addition, minimising the size of the sensor also reduces its influence on the THMC performance of, in this case, a bentonite block. Finally, the power consumed by a single traditional sensor varies between $1 \mathrm{~mW}$ and $1 \mathrm{~W}$, which inevitably reduces the battery life for wireless transmission and hampers the longevity of sensors in enclosed deposition galleries. 
MEMS sensors are a potential alternative to traditional sensors due to their significantly reduced size and lower power consumption. MEMS sensors are fabricated using micro-machining technology in the semiconductor industry and are common in applications such as the automotive industry. The typical dimension of a MEMS sensor is at a micrometric scale for the sensor itself, and around 2$3 \mathrm{~mm}$ for the accompanying exterior packaging. MEMS sensors convert physical quantities to electrical signals based on different physical transduction effects such as piezoelectricity and piezoresistivity. In comparison with macro-scale traditional sensors, MEMS sensors provide higher measurement accuracy, improved spatial resolution in a limited space, and a longer life cycle resulting from low power consumption in the order of microwatts (Akyildiz et al. 2002). The deployment of MEMS sensors is also a cost-effective solution for many engineering applications thanks to the decreasing unit price of MEMS sensors under mass fabrication.

As the development of the MEMS technology has only burgeoned in the past 20 years thanks to the exponential growth in microelectronics industry, the application of MEMS sensors in GDFs and other civil engineering projects still faces several key challenges in the engineering field (Ceylan et al. 2011). First of all, as the selection and coordination of MEMS sensors for various THM properties are more application-specific than generic, custom designed multi-sensor monitoring system will have an advantage over the product-specific commercial sensor devices available in the market, which require extensive and interdisciplinary research input. Another key issue is the survivability of MEMS sensors and the associated electronics in geological materials with significantly larger dimensions. Effective encapsulation of MEMS sensors is thus crucial to ensure robust long-term 
performance in harsh geological monitoring environments. In addition, adapted calibration methods of the MEMS sensors need to be developed, as the sensors embedded inside the monitored barriers, once emplaced, are often physically inaccessible from the testing environment. Further challenges including the field testing of MEMS sensors in the EBS and the wireless signal transmission and power supply will also attract continuous interest in our future research activities.

\section{Design of the Multi-sensor Monitoring System}

In response to the main challenges mentioned above for the application of MEMS sensors in GDFs, the first prototype of a multi-sensor monitoring system has been implemented for its application in compacted bentonite. Both high-precision temperature and relative humidity (RH) MEMS sensors are integrated into a single monitoring device. The key factors considered for the selection of the sensors were the sensor accuracy, sensor volume, power consumption, working temperature range and communication protocol with the control system. After comparing a number of high-end commercial MEMS sensors with regard to the criteria mentioned above, the Maxim $^{\circledR} 31725$ temperature sensor and the Sensirion ${ }^{\circledR}$ SHT25 relative humidity sensor were selected. The Maxim ${ }^{\circledR} 31725$ (Maxim, 2013) temperature sensor has a typical precision of $\pm 0.5^{\circ} \mathrm{C}$ for a measurement range between $-55^{\circ} \mathrm{C}$ and $150^{\circ} \mathrm{C}$, and the Sensirion ${ }^{\circledR}$ SHT25 (Sensirion, 2014) RH sensor has a precision level of $\pm 1.8 \%$ within the $10 \% \sim 90 \% \mathrm{RH}$ range, and $\pm 3 \%$ within the full RH range. Both sensors have a dimension of $3 \mathrm{~mm} \times 3 \mathrm{~mm} \times 1 \mathrm{~mm}$ and can be integrated onto a single printed circuit board (PCB). The temperature within the EBS is expected to vary between $0^{\circ} \mathrm{C}$ and $100^{\circ} \mathrm{C}$ from the host rock to the canister 
(Juvankoski et al. 2012), and the RH within the EBS is expected to vary between $20 \%$ (bentonite at initial compacted state) and $100 \%$ (in case of bentonite saturation). Therefore, the measurement ranges of the sensors cover the entire anticipated temperature and RH variation ranges within the EBS.

In order to reduce the intrusiveness of the sensor block into the bentonite sample, a method of hierarchical design has been adopted by connecting a number of single sensor blocks to an upstream controller board (motherboard), as illustrated in Fig. 2a. The sensor block, which would be emplaced within the bentonite, is only equipped with the sensor and its connector, while all other functional components have been integrated onto a motherboard that can be installed outside the bentonite barrier. The power supply and signal transmission between the sensors and the motherboard are established by the use of heat-resistant PTFE-coated wires that are compatible with environmental temperatures between $-60^{\circ} \mathrm{C}$ and $200^{\circ} \mathrm{C}$. These have the potential to be replaced in the future with a wireless transmission system. The size of a single sensor board for installation in the bentonite barrier is $9 \mathrm{~mm} \mathrm{x}$ $11 \mathrm{~mm}$, which will assure minimum impact of the sensor block on the overall hydro-mechanical behaviour of the bentonite.

For the sake of programming conformity, the temperature sensor Maxim ${ }^{\circledR} 31725$ and the RH sensor Sensirion ${ }^{\circledR}$ SHT25 both generate a digital signal output and communicate with the microcontroller via the $\mathrm{I}^{2} \mathrm{C}(\mathrm{NXP}, 2014)$ serial bus protocol. As a result, it is possible to programme the microcontroller in order to use a single reconfigurable motherboard connected to multiple temperature and $\mathrm{RH}$ sensors for simultaneous data collection and display. The total number of sensors hosted on a single motherboard is eight in our current design, but can be increased if required 
for future applications. The controller board is also compatible with other types of digital sensors communicating via the $\mathrm{I}^{2} \mathrm{C}$ protocol, which makes the system highly flexible for incorporating additional sensor types in the event of comprehensive and wide-ranging monitoring needs.

The temperature and $\mathrm{RH}$ values measured by the sensors are sent by the microcontroller to a computer via the RS232 serial communication port. A bespoke interactive user interface has been developed in Labview ${ }^{\circledR}$ (Fig. 2b) that enables the real-time graphical display of temperature and $\mathrm{RH}$ from all sensors, as well as data storage on hard disk for further analysis. The interface also allows the user to activate/deactivate individual sensors in the monitoring system and to control their measurement frequency and duration.

\section{Sensor Encapsulation}

As the temperature and $\mathrm{RH}$ sensors will be emplaced within hydrated bentonite blocks, and will therefore be in direct contact with hydrated bentonite during the experiment, it is important to encapsulate the sensor and other electronic devices. Effective encapsulation is required to protect the sensor, and other electronic components, from moisture, chemical corrosion and exterior mechanical shocks. The temperature sensor can be fully encapsulated in a thermally conductive material. In the case of the RH sensor SHT25, however, the sensor encapsulation is more challenging, as the sensing area of the relative humidity must be exposed to the measuring environment (Fig. 3a). Moreover, whilst the sensing area must remain unshielded in order to measure the $\mathrm{RH}$, the area should not be directly immersed in water (e.g. due to bentonite saturation), nor can particles be allowed to 
block the sensing area, which would significantly hamper the accuracy of the sensor. In order to avoid direct contact between the sensor and the bentonite, a filter cap designed by Sensirion ${ }^{\circledR}$ (Sensirion, 2011) was incorporated to cover the RH sensor on the PCB board prior to encapsulation. The filter provides better protection of the sensor against mechanical impact and contamination, whilst still allowing the propagation of water molecules between the measuring environment and the RH sensor through the PTFE filter membrane. Extra caution is thus required during the encapsulation process so as not to cover the filter membrane area with the sealant.

Two encapsulation methods were tested here and their encapsulation effects are compared. The first method is named conformal coating (Chao et al.2012), which is achieved by applying a spray aerosol onto the surface of the PCB sensor block. The spray is composed of dedicated organic compounds (xylene, hydrocarbons, etc.) and can quickly coagulate to form a thin colloidal film on the surface of the sensor board. The conformal coating process is easy to operate, but the encapsulation quality was compromised after weeks of exposure to a high humidity environment. This occurred because the thin colloidal layer only delays the ingress of water to the surface of PCB board and does not completely prevent it (Tencer $e t$ al. 2002). Fig. 3b shows a conformal coated sensor after two weeks' exposure to a high RH environment ( 90\%), oxidized copper wires and solder joints cover a considerable proportion the sensor board surface, which rendered the sensor block dysfunctional.

The second encapsulation technique trialled was the recently developed 'potting' method (Chao et al.2012) that uses polyurethane resin as an encapsulation material. 
In this technique, a specifically-designed silicone rubber mould must be manufactured for sensor board encapsulation. The rubber mould designed had a rectangular void with sufficient size to fit in the sensor board and was accessible from the top surface of the rubber. The sensor board was then installed in the hollow area and soaked in polyurethane resin. The resin volume was carefully controlled to avoid covering the filter cap, as described above. After the curing process, which was assisted by a hardener, the silicone mould could be 'peeledoff', leaving a rectangular polyurethane block that enclosed the sensor board inside. As indicated in Fig. 3c, the encapsulated sensor block has similar dimensions to the sensor board before encapsulation. This 'potting' method of encapsulation proved robust; the encapsulated sensors were stable and resistive to the influence of high RH throughout all of the following experiments, over the test periods of several weeks.

\section{System Testing and Calibration}

The functionality and accuracy of the multiple sensors were tested using several different methods. The Maxim ${ }^{\circledR} 31725$ temperature sensor was tested inside a controlled climatic chamber together with a high-precision Fluke ${ }^{\circledR} 5624$ Platinum resistance thermometer (PRT) probe, which served as a reference probe. The temperature inside the climatic chamber was cycled between $-20^{\circ} \mathrm{C}$ and $120^{\circ} \mathrm{C}$. Fig. $4 \mathrm{c}$ shows the temperature measurements from four Maxim $^{\circledR} 31725$ temperature sensors ( 2 exposed sensors and 2 encapsulated sensors) and the PRT probe during the cooling process, where temperature fell gradually from $120^{\circ} \mathrm{C}$ to $20^{\circ} \mathrm{C}$. The discrepancy of measured temperatures among different sensors and the 
PRT probe are within $\pm 0.5^{\circ} \mathrm{C}$ for the temperature range between $20^{\circ} \mathrm{C}$ and $100^{\circ} \mathrm{C}$, and up to $1.5^{\circ} \mathrm{C}$ for higher temperature, which provides a satisfactory accuracy level in comparison with most temperature sensors used for monitoring in underground GDFs for radioactive waste disposal applications (AITEMIN, 2013). The performance of sensors in the temperature range between $-20^{\circ} \mathrm{C}$ and $20^{\circ} \mathrm{C}$ could not be assessed against the PRT probe because this range is not covered by the PRT probe. However, the temperatures measured by all 4 temperature sensors are consistent with discrepancies of less than $1^{\circ} \mathrm{C}$.

The Sensirion ${ }^{\circledR}$ SHT25 RH sensor was tested in enclosed containers (Fig. 4a) by exposure to saturated salt solutions at different temperatures. RH values imposed by salt solutions were derived from Greenspan (1977) and Winston et al. (1960). The first test involved exposure of RH sensors to salt solutions at room temperature $\left(20^{\circ} \mathrm{C}\right)$. The $\mathrm{RH}$ sensor readings were registered after the equilibrium of $\mathrm{RH}$ inside the containers was achieved, which took 2 to 3 hours depending on the size of the container. Table 1 shows the RH values imposed by the salt solutions and measured by the RH sensors. Error in the RH range from $10.2 \%$ to $64.3 \%$ remains within the range $\pm 1.8 \%$, which is the accuracy specified by the supplier. The error increases with increasing $\mathrm{RH}$ to a maximum error of $5.4 \%$ for an imposed $\mathrm{RH}$ of 98\%. The limitation in the accuracy of RH sensors at high humidity levels is quite common among contemporary RH sensors for engineering applications.

Fig. 4b shows the RH measurements returned by the sensors against the RH values imposed by the salt solutions. Data points lie on the 1:1 line in the low and medium RH range and deviate consistently from the 1:1 line at high RH values. Accuracy of the sensor can be improved by introducing a calibration model to the sensor 
reading. If $\mathrm{RH}_{\mathrm{T}}$ denotes the imposed $\mathrm{RH}$ values of saturated solutions, and the $\mathrm{RH}$ value returned by the sensor, $\mathrm{RH}_{\mathrm{S}}$, is interpreted as a 'raw' reading, a new calibration function can be derived. A quadratic polynomial equation can be established which fits adequately the calibration data points (Fig. 4b):

$\mathrm{RH}_{\mathrm{S}}=0.001461\left(\mathrm{RH}_{\mathrm{T}}\right)^{2}+0.8912 \mathrm{RH}_{\mathrm{T}}+2.124$

Using the calibration function above, it is possible to calculate a corrected $\mathrm{RH}$ of saturated solutions associated with calibration curve (1), and the error in the measurement is shown in Table 1. It can be seen that accuracy in the high RH range can be significantly improved by using the calibration curve (1), which returns a standard deviation of the error of $0.8 \%$.

\begin{tabular}{|c|c|c|c|c|c|}
\hline Solution & $\begin{array}{c}\text { Imposed } \mathrm{RH} \\
\text { value }\end{array}$ & $\begin{array}{c}\mathrm{RH} \text { value read } \\
\text { by the sensor }\end{array}$ & Error & $\begin{array}{c}\text { Corrected } \\
\mathrm{RH} \text { value }\end{array}$ & Error \\
\hline $\mathrm{KOH}$ & $10.2 \%$ & $11.2 \%$ & $1.0 \%$ & $10.0 \%$ & $0.2 \%$ \\
\hline $\mathrm{Nal}$ & $39.6 \%$ & $40.4 \%$ & $0.8 \%$ & $40.3 \%$ & $0.7 \%$ \\
\hline $\mathrm{Na} 2 \mathrm{Cr}_{2} \mathrm{O} 7$ & $54.5 \%$ & $56.0 \%$ & $1.5 \%$ & $55.4 \%$ & $0.9 \%$ \\
\hline $\mathrm{NH}_{4} \mathrm{NO}_{3}$ & $64.3 \%$ & $63.0 \%$ & $1.3 \%$ & $62.0 \%$ & $2.3 \%$ \\
\hline $\mathrm{NaCl}$ & $75.5 \%$ & $77.8 \%$ & $2.3 \%$ & $75.5 \%$ & $0.0 \%$ \\
\hline $\mathrm{NH} 4 \mathrm{Cl}$ & $78.6 \%$ & $82.4 \%$ & $3.8 \%$ & $79.7 \%$ & $1.1 \%$ \\
\hline $\mathrm{KCl}$ & $85.1 \%$ & $88.1 \%$ & $3.0 \%$ & $84.7 \%$ & $0.4 \%$ \\
\hline $\mathrm{BaCl} \%$ & $90.0 \%$ & $94.4 \%$ & $4.4 \%$ & $90.2 \%$ & $0.2 \%$ \\
\hline $\mathrm{CuSO} 4$ & $98.0 \%$ & $103.4 \%$ & $5.4 \%$ & $97.9 \%$ & $0.1 \%$ \\
\hline
\end{tabular}

Table 1 Comparison of theoretical, measured and corrected $\mathrm{RH}$ of saturated salt solutions at room temperature

A coupled measurement of temperature and RH has been carried out by placing the containers with the sensor suspended above the saturated salt solution in a temperature-controlled climatic chamber. Inside each container, the SHT25 RH 
sensor measures simultaneously the temperature and RH imposed by the saturated solution. The temperature inside the climatic chamber was programmed to increase from $20^{\circ} \mathrm{C}$ to $60^{\circ} \mathrm{C}$ in steps of $10^{\circ} \mathrm{C}$. As the solubility of salts usually increases with temperature, the $\mathrm{RH}$ inside the containers is anticipated to decrease during the heating process. This is confirmed by the experimental data provided by Greenspan (1977) and Winston et al. (1960). For this experiment, four solutions with a higher temperature coefficient of $\mathrm{RH}$ variation were selected: $\mathrm{KOH}, \mathrm{NaI}, \mathrm{Na}_{2} \mathrm{Cr}_{2} \mathrm{O}_{7}$ and $\mathrm{KCl}$. Temperature in each step was maintained for 48 hours and only the data acquired after stabilisation of the signal were taken into account.

Fig. 4d presents the $\mathrm{RH}$ values read by the sensors exposed to the four salt solutions at different temperatures. The imposed RH values according to Greenspan (1977) and Winston et al. (1960) are also plotted on the same figure.

The RH values read by the sensor appear to match favourably the imposed values of $\mathrm{RH}$ at $20^{\circ} \mathrm{C}$. As temperature increases, a different response is observed in the low, medium and high $\mathrm{RH}$ range. In the low $\mathrm{RH}$ range $(\mathrm{KOH})$, the $\mathrm{RH}$ values read by the sensor deviated from the ones imposed by the salt solution although error remains within the range of $4 \%$. This error can be considered acceptable in soil moisture measurement: in the very low humidity range, a variation of $4 \%$ in $\mathrm{RH}$ has little effect on hydro-mechanical response of clays and high accuracy in the measurement of RH is therefore not required.

In the medium range of $\mathrm{RH}(\mathrm{NaI})$, the $\mathrm{RH}$ values read by the sensor compare favourably with the imposed value. In the high $\mathrm{RH}$ range $\left(\mathrm{Na}_{2} \mathrm{Cr}_{2} \mathrm{O}_{7}\right.$ and $\left.\mathrm{KCl}\right)$, however, the RH values read by the sensor deviate more significantly from the 
ones imposed by the salt solution as the temperature increases. At a temperature of $50^{\circ} \mathrm{C}$, the error is about $\Delta \mathrm{RH}=7 \%$ for the $\mathrm{Na}_{2} \mathrm{Cr}_{2} \mathrm{O}_{7}$ and $\Delta \mathrm{RH}=5.6 \%$ for the $\mathrm{KCl}$. This result indicates that temperature has also a significant influence on the accuracy of the RH sensor. The error of 5-7\% at high RHs has significant effects on the hydro-mechanical response of clays and hence a high accuracy in the measurement of $\mathrm{RH}$ is required in this range.

In summary, this experiment verifies the coupled monitoring capability of the RH sensor with a compromised accuracy at high temperatures in the high RH range. To use the MEMS sensors in engineered barrier systems, a temperature-dependent calibration curve should therefore be developed. This work is currently underway.

\section{Conclusions and Perspectives}

A MEMS-sensor-based (Micro-Electro-Mechanical System) monitoring device has been developed and then verified within a controlled thermal-hydro environment. The system integrates state-of-the-art temperature and RH MEMS sensors onto a reconfigurable interface circuit, with a dedicated software user interface. The new device has several advantages: (1) the size of the sensor block is highly reduced to minimise intrusiveness into the bentonite sample and to provide increased spatial accuracy by comparison to other contemporary sensors; (2) the reconfigurable interface circuit design means that multiple sensors (up to 50) can be connected to a single motherboard removing the requirement for multiple data-loggers; (3) the sensors are encapsulated in polyurethane to enhance their resistivity to corrosion and to mechanical stress (4) verification tests have shown the sensors to have excellent measurement accuracy in an extended working temperature range and a 
new calibration model for the RH sensor has been developed to counterbalance its offset in the high RH measurement range.

The primary limitation of the current sensor monitoring system is the use of wires, which increases the complexity of sensor installation. The long-term use of wires, for operational monitoring in a repository, may also compromise the engineered barrier by providing pathways along which radionuclides could migrate. Future research will focus on the integration of a wireless communication module and a power supply module into the encapsulated sensor block, thus enabling accurate long-term environmental monitoring within the engineered barrier system of a geological disposal facility. Other issues with respect to the incorporation of MEMS sensors in the bentonite barrier, such as the influence of MEMS sensors on the functionality and THMC properties of bentonite, will also be investigated.

\section{Acknowledgement}

We would like to acknowledge the Engineering and Physical Sciences Research Council (EPSRC) and Radioactive Waste Management Limited (RWM) for their financial support in the framework of the SAFE Barriers Project. 


\section{References}

AITEMIN (2013) State of art report on monitoring technology. Modern Project WP2 report, 2013

Akyildiz, I. F., Su, W., Sankarasubramaniam, Y. and Cayirci, E. (2002) Wireless sensor networks: a survey, Computer Networks, 38, 393-422

Alonso, E. E., Springman, S. M. and Ng, W. W. (2009) Monitoring large-scale tests for nuclear waste disposal, Geotechnical and Geological Engineering, 26, No. $6,817-826$

Breen, B. J., Garcia-Sineriz, J. L., Maurer, H., Mayer, S., Schröder, T. J. and Verstricht, J. (2012) EC MoDeRn Project: In-situ demonstration of innovative monitoring technologies for geological disposal, WM2012 Conference

Ceylan H. , Gopalakrishnan, K., Taylor, P., Shrotriya, P., Kim, S., Prokudin, M., Wang, S., Buss, A. F. and Zhang, J. et al., (2011) A feasibility study on embedded micro-electromechanical sensors and systems (MEMS) for monitoring highway structures, Iowa highway research board report No. TR-575

Chao, N. H., Dispenza, J. A. and DeAngelis, M. E. (2012) Encapsulating protective layers for enhancing survivability of circuit board assemblies in harsh and extreme environments, ASME 2012 International Mechanical Engineering Congress and Exposition, 9, 363-373

Greenspan, L. (1977) Humidity fixed points of binary aqueous solutions, Journal of research of the national bureau of standards, 81A, No. 1, 89-96 
Juvankoski, M., Ikonen, K., and Jalonen, T. (2012) Buffer production line 2012: Design, production and initial state of the buffer. Posiva report 2012-17

Lidskog, R. and Andersson, A.C. (2002) The management of radioactive waste: a description of ten countries, SKB report, ISBN 91-973987-3-X

Maxim $^{\circledR} 31725$ datasheet. Ver.2013. Available from: http://datasheets.maximintegrated.com/en/ds/MAX31725-MAX31726.pdf $\mathrm{NXP}^{\circledR}$ (2014) I ${ }^{2} \mathrm{C}$-bus specification and user manual. Rev. 6, 2014. Available from: http://www.nxp.com/documents/user_manual/UM10204.pdf

Tencer, M. and Moss, J. S. (2002) Humidity management of outdoor electronic equipment: methods, pitfalls, and recommendations. IEEE Transactions on Components and Packaging Technologies, 25(1), 66-72

Sensirion ${ }^{\circledR}$ Filter Cap SF2 datasheet. Ver.2011. Available from: http://www.sensirion.com/sf2/

Sensirion ${ }^{\circledR}$ SHT25 datasheet. Ver.2014. Available from: http://www.sensirion.com/fileadmin/user_upload/customers/sensirion/Dokumente/ Humidity/Sensirion_Humidity_SHT25_Datasheet_V3.pdf Winston, P. W. and Bates, D. H. (1960) Saturated solutions for the control of humidity in biological research, Ecology, 41, Issue 1, 232-237 
Figures

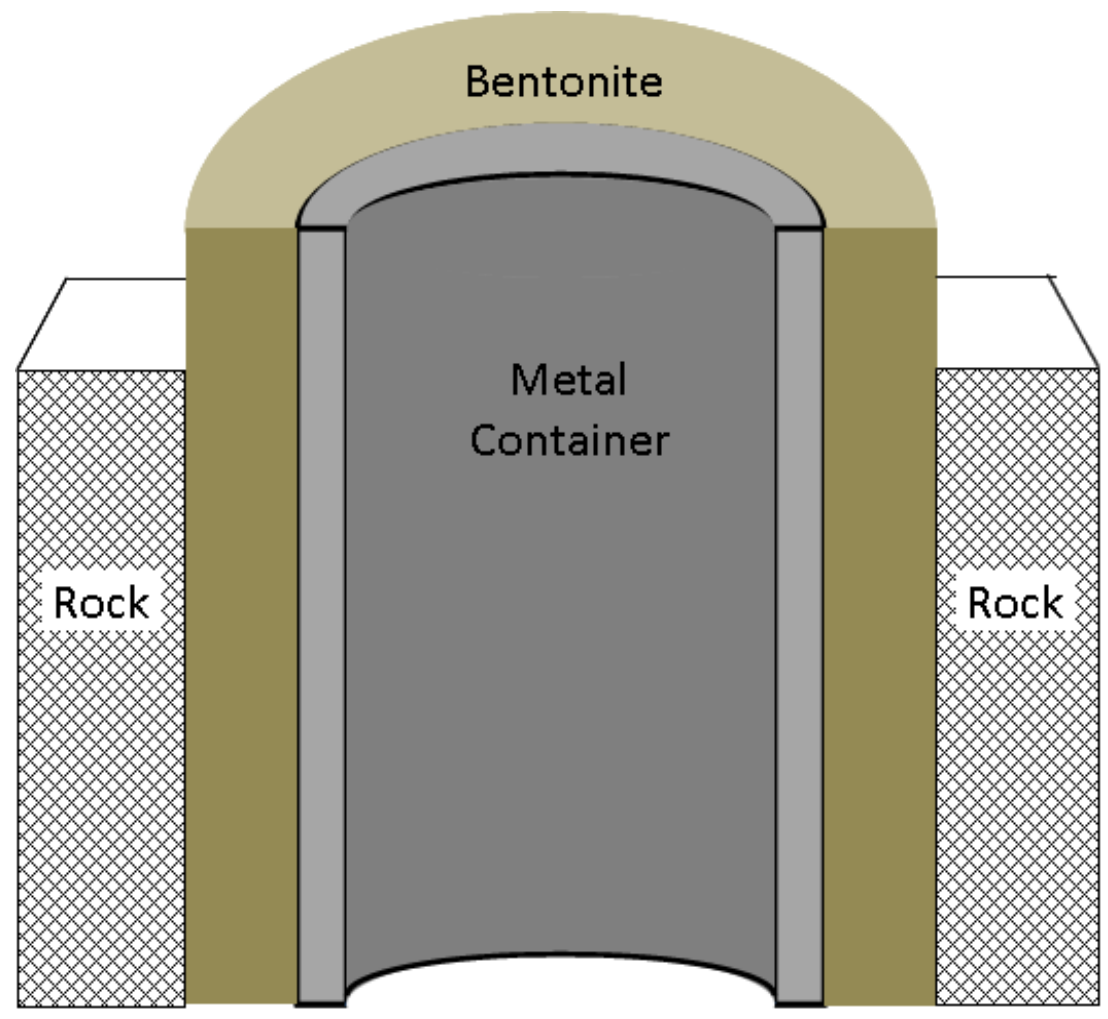

Fig. 1 Schematic cross-section through bentonite engineered barrier system 


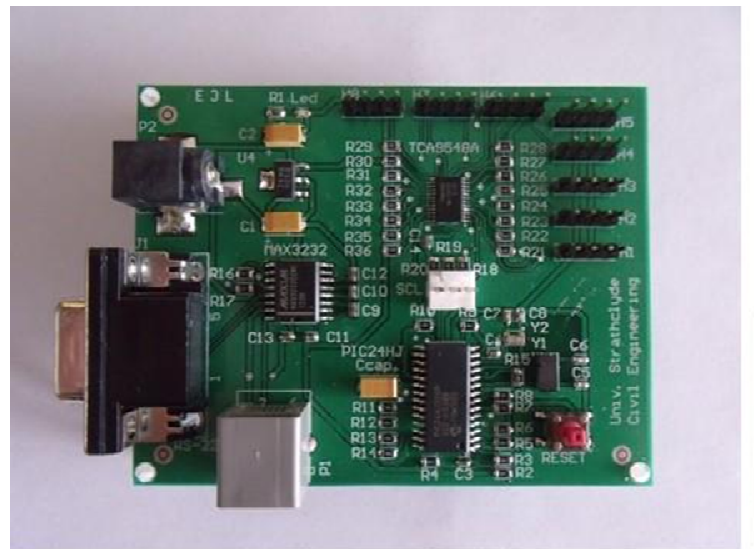

Controller board
Sensor

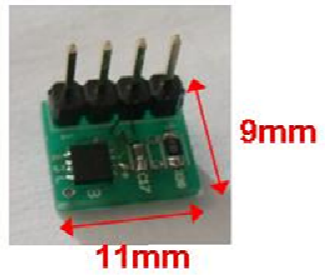

(a)

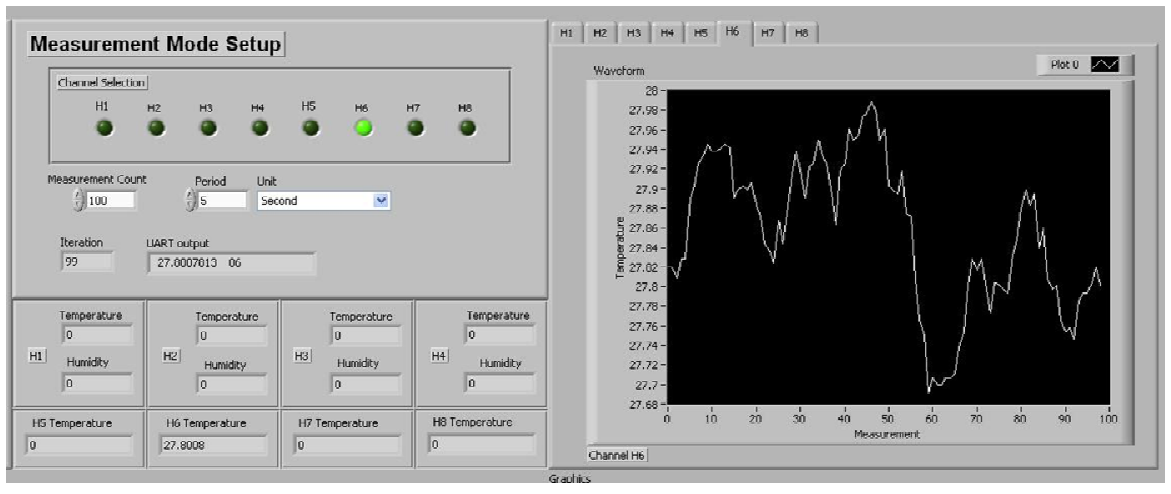

(b)

Fig. 2 (a) PCB-based controller board and sensor board (prior to encapsulation); (b) interactive user interface and real-time graphics demonstration in Labview 


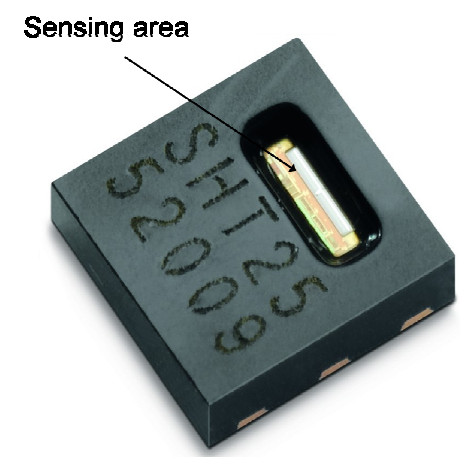

(a)

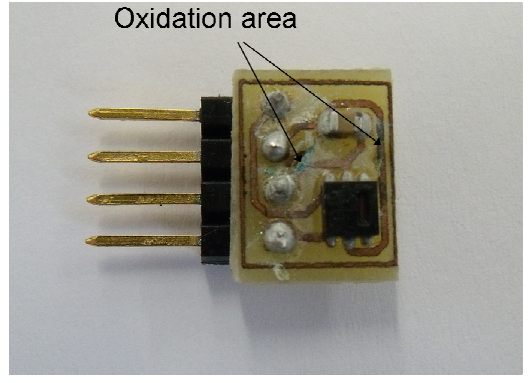

(b)

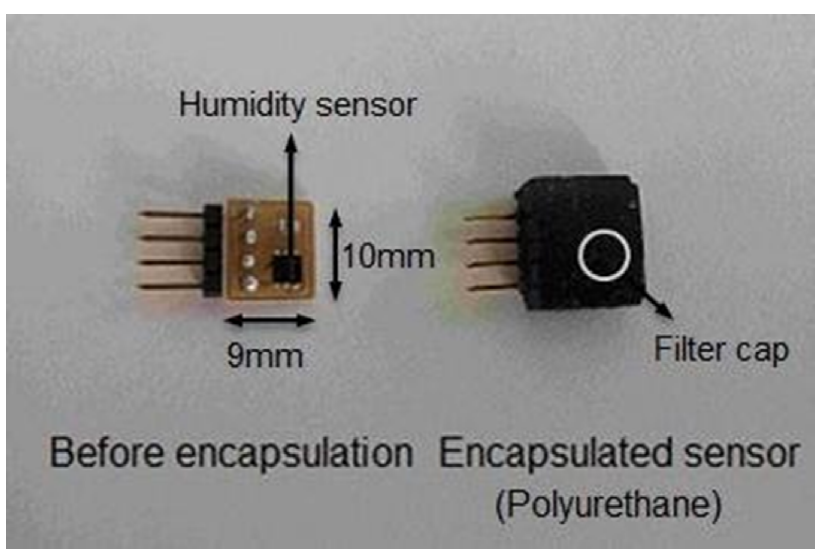

(c)

Fig. 3 (a) enlarged photo of the Sensirion SHT25 sensor (source: product website); (b) sensor board oxidation after 2-week exposure to humid environment; (c) SHT25 sensor board before and after encapsulation 


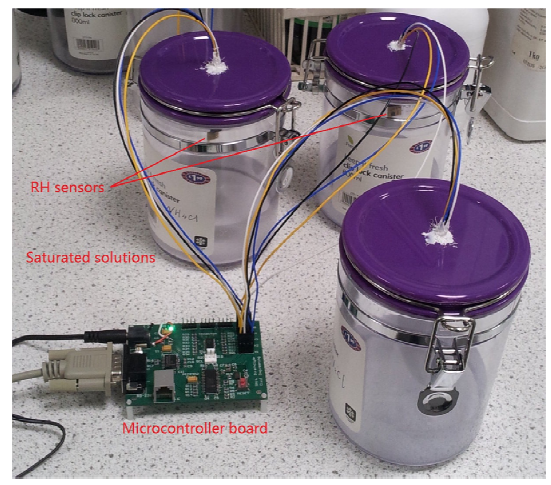

(a)

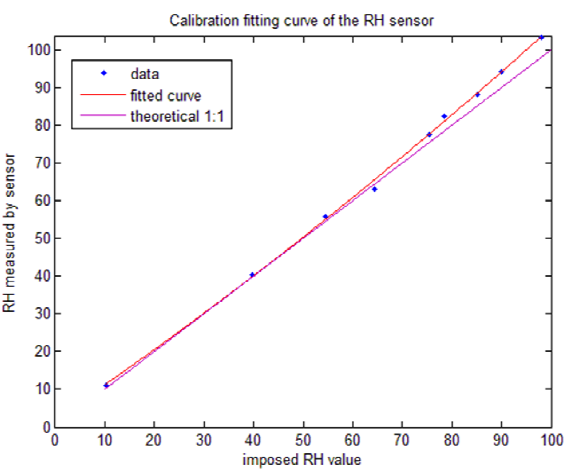

(b)
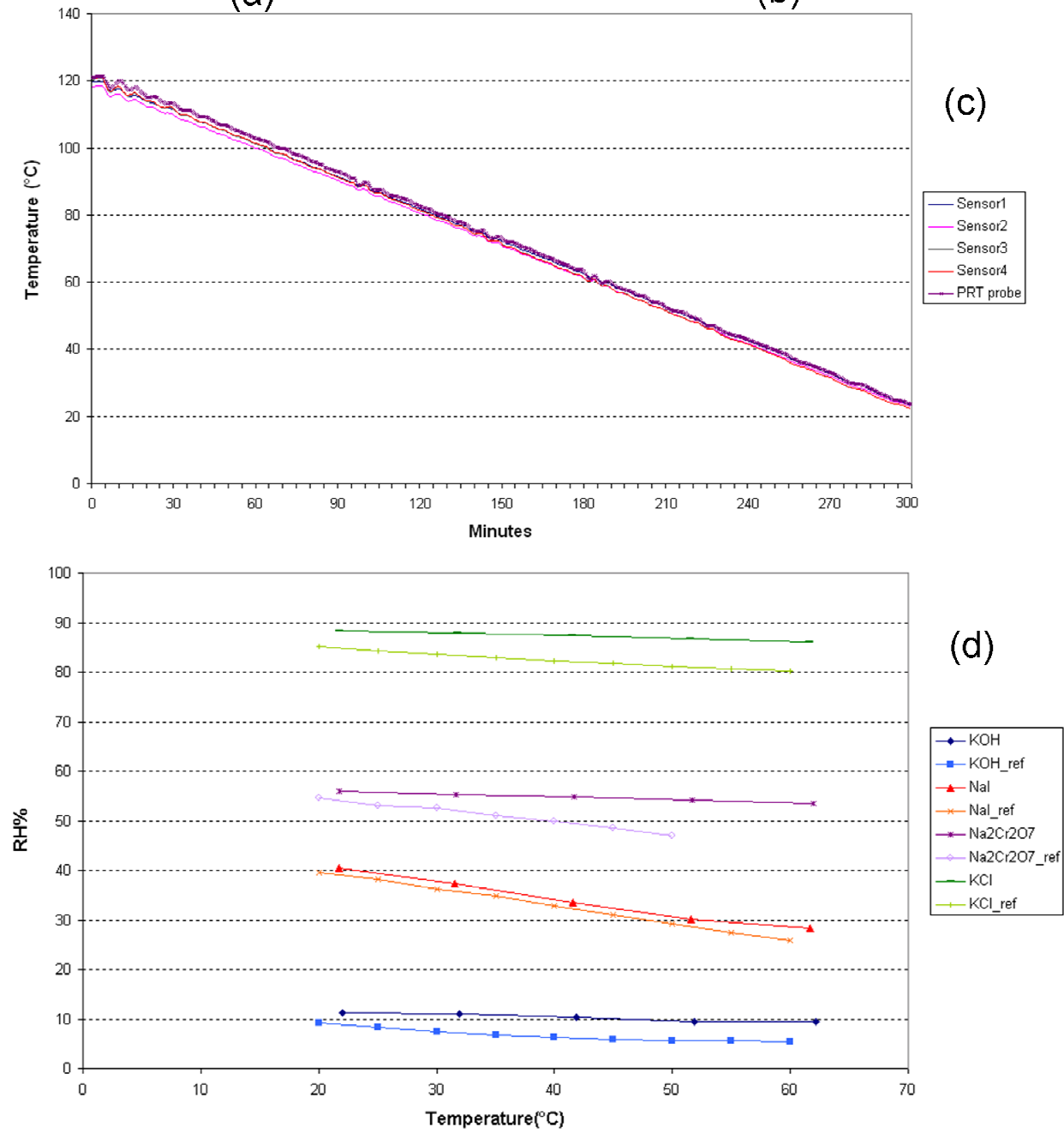

Fig. 4 (a) RH sensor measurement above saturated solutions in enclosed containers; (b) fitting curve of the calibration model for the RH sensor; (c) temperature measured by sensors and PRT probe in climatic chamber cooling process; (d) RH measurements under increasing environment temperature 